\title{
58. The Difference in the Self-reversal of Ne-lines excited by Direct and Alternate Currents. (II)
}

\author{
By Hantaro Nagaoka and Tadao Mishima. \\ Institute of Physical and Chemical Research.
}

(Rec. April 30, 1931. Comm. by H. NagaoKA, M.I.A., May 12, 1931.)

On further examination of the difference in the reversal of $\mathrm{Ne}$ lines ${ }^{1)}$ excited by a.c. and d.c., the amount was found to depend largely on the current density. Using a tube of $1.5 \mathrm{~mm}$. bore, the same point of the luminous column at the mouth of the capillary of a Ne-tube cooled in liquid air was examined by exciting it with terminal voltage of $1200 \mathrm{v}$ for a.c. and d.c. The a.c. was rectified by a thyratron, so that the current was intermittent.

By adjusting the exciting current, the variation of the self-reversal with current was studied. From the smallness of the phenomenon, it is difficult to speak definitely about the complex nature of the phenomenon. One fact is, however, certain that the reversal in the lines belonging to $\left(s_{2}, p\right)$ is deviated from other groups. The curves for a.c. and d.c. are plotted for 5852 and 6678 (Fig. 1 and 2). They show that for weak excitation, the reversal is greater for d.c. than for a.c., and for stronger excitation, the d.c. curve lies below that for a.c.. The point of crossing is different for the lines. The difference, however, is of the order of a few $m \AA$ 's, so that the exact value is uncertain.

For $\left(s_{3}, s_{4}, s_{5} ; p\right)$ lines, there is still some difference but it is not so distinct as for $\left(s_{2}, p\right)$ lines. The general tendency is to reach a maximum and then gradually to diminish with increase of the current. An instance is given for 5882 (Fig. 3). Considering the smallness of the amount and the vagueness of the photograms, exact measurements will reveal differences with the construction of the tube, the pressure, and the temperature of the gas.

1) Nagaoka and Mishima: Proc. 7 (1931). 


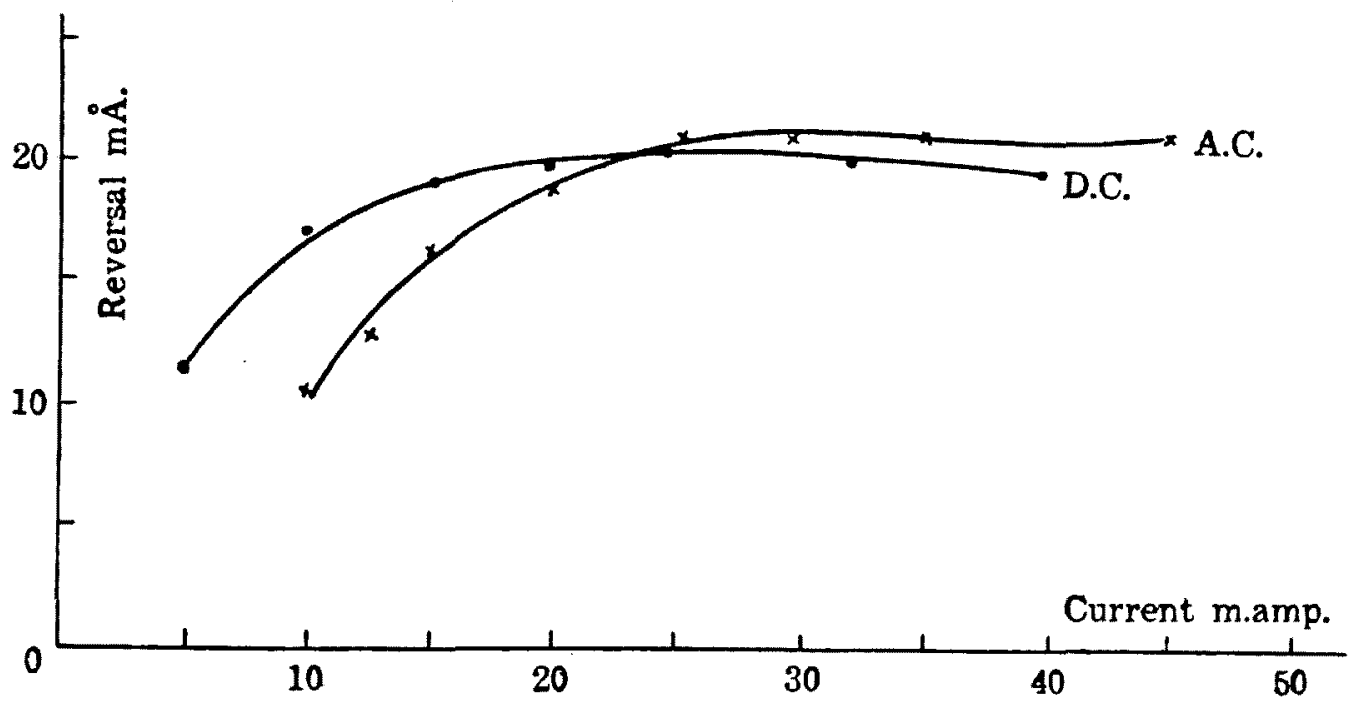

Fig. 1. $\lambda 5852\left(1 s_{2}-2 p_{1}\right)$.

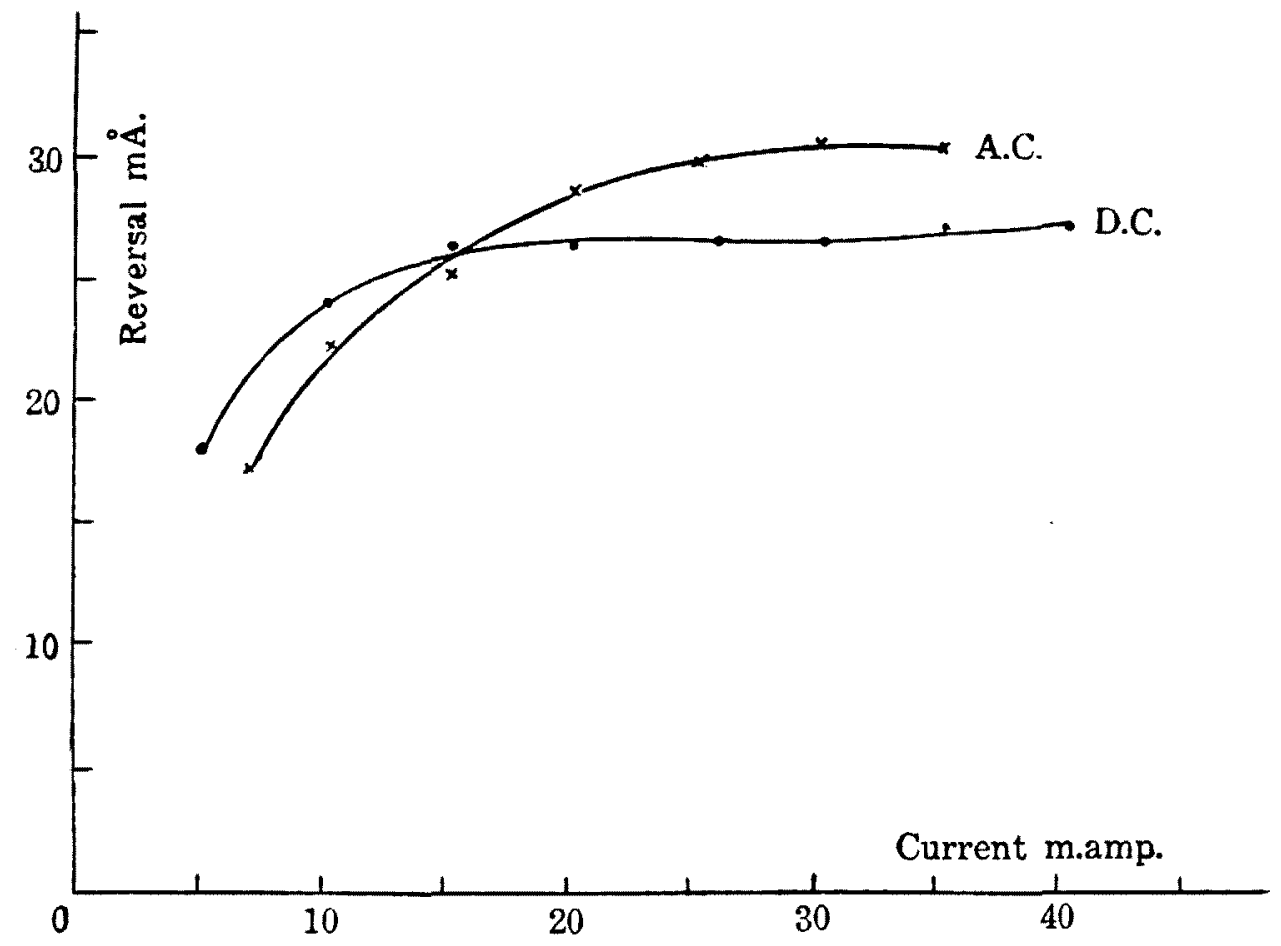

Fig. 2. $\lambda 6678\left(1 s_{2}-2 p_{4}\right)$. 


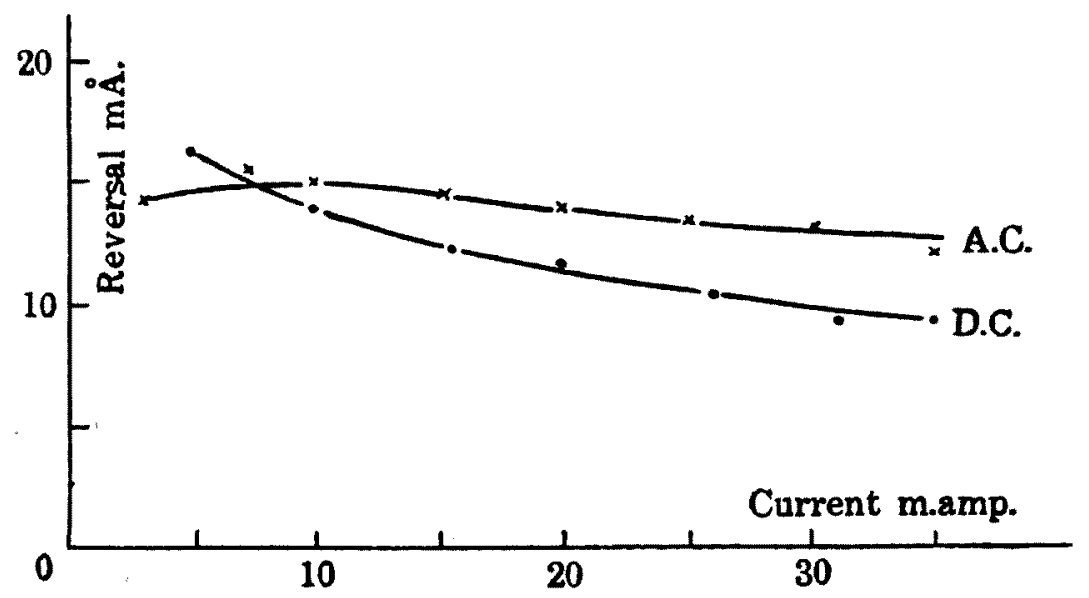

Fig. 3. $\lambda 5882\left(1 s_{5}-2 p_{2}\right)$. 